\title{
Fatores associados à Síndrome Respiratória Aguda Grave em uma Região Central do Brasil
}

\author{
Factors associated with Severe Acute Respiratory Syndrome \\ in a Brazilian central region
}

Kamilla Lelis Rodrigues de Araujo (https://orcid.org/0000-0002-0090-358X) ${ }^{1}$

Érika Carvalho de Aquino (https://orcid.org/0000-0002-5659-0308) ${ }^{2}$

Lara Lívia Santos da Silva (https://orcid.org/0000-0002-8948-7884) ${ }^{2}$

Yves Mauro Fernandes Ternes (https://orcid.org/0000-0002-6240-3054) ${ }^{2}$
${ }^{1}$ Programa de PósGraduação em Assistência e Avaliação em Saúde, Universidade Federal de Goiás (UFG). Av. Rúda Quadra 41A, Lote 10, Vila Brasília. 74905-760 Aparecida de Goiânia GO Brasil. kamillalelis@hotmail.com ${ }^{2}$ Instituto de Patologia Tropical e Saúde Pública, Departamento de Saúde Coletiva, UFG. Goiânia GO Brasil.

\begin{abstract}
Severe Acute Respiratory Infection (SARI) is a notifiable syndrome that must be investigated. This study aimed to analyze the epidemiological profile and factors associated with SARI-related hospitalization and deaths reported in Goiás. Retrospective cohort study, with data from the investigation files of the Notifiable Diseases Information System's Influenza Web. Multivariate analysis methods were employed to verify the association between exposure variables with the outcomes of ICU admission and death. A total of 4,832 SARI cases were reported in Goiás from 2013 to 2018. The primary etiological diagnosis was Influenza A (22.3\%) with the predominant subtype A (H1N1pdm09), followed by the Respiratory Syncytial Virus. A total of $34.6 \%$ of the patients required ICU admission, and 19\% died. A longer time to start treatment with antivirals was associated with a higher likelihood to have an ICU admission, while a previous non-vaccination against Influenza, longer time to start treatment, and older age were associated with a higher likelihood to suffer death. The study showed a high frequency of respiratory diseases caused by the Influenza virus in Goiás and that the severity of the syndrome, characterized by ICU admission and deaths, is associated with the start of antiviral treatment vaccine status, and patient's age.

Key words Human Influenza, Surveillance, Epidemiology, Severe Acute Respiratory Infection
\end{abstract}

Resumo A Sindrome Respiratória Aguda Grave (SRAG) deve ser notificada e investigada. O objetivo do estudo foi analisar o perfil epidemiológico e fatores associados à internação e óbitos por SRAG notificados em Goiás. Estudo de coorte retrospectiva, com dados das fichas de investigação do Sistema de Informação de Agravos de Notificação Influenza Web. Métodos de análise multivariada foram realizados para verificar a associação entre variáveis de exposição com os desfechos internação em UTI e óbito. Entre 2013 e 2018 foram notificados 4.832 casos de SRAG em Goiás. O principal diagnóstico etiológico foi influenza $A(22,3 \%)$ com o subtipo A (H1N1pdm09) predominante, seguido pelo Vírus Sincicial Respiratório. 34,6\% dos pacientes necessitaram de internação em UTI e 19\% evoluíram para o óbito. Maior tempo de início do tratamento com antiviral foi associado à maior chance de internação em UTI, enquanto a não vacinação prévia contra a influenza, maior tempo para início do antiviral e idade mais avançada foram associados à maior chance de óbito. O estudo mostrou uma elevada frequência de doenças respiratórias provocadas pelo vírus Influenza no estado de Goiás e que a gravidade da sindrome, caracterizada pela internação em UTI e óbitos, está associada com o tempo de início do tratamento com o antiviral, o status vacinal e a idade do paciente.

Palavras-chave Influenza Humana, Vigilância, Epidemiologia, Sindrome Respiratória Aguda Grave 


\section{Introdução}

A Síndrome Respiratória Aguda Grave (SRAG) é uma síndrome respiratória viral infecciosa causada por vírus influenza e outros agentes etiológicos, como o vírus sincicial respiratório (VSR), parainfluenza e adenovírus, que infectam o trato respiratório superior ${ }^{1}$. Os pacientes apresentam um quadro gripal associado à dispneia ou taquipneia (frequência respiratória igual ou acima de 20 incursões por minuto) ou hipoxemia, com saturação de oxigênio (SpO2) < 95\% em ar ambiente ${ }^{2}$.

Trata-se de uma doença de importância epidemiológica que deve ser notificada e investigada. O monitoramento epidemiológico de SRAG no Brasil ocorre por meio da vigilância epidemiológica da doença por meio do preenchimento das fichas de notificação, através do Sistema de Informação de Agravos de Notificação (Sinan), e funcionamento das unidades sentinelas. Essas unidades funcionam monitorando os casos hospitalizados e óbitos por SRAG, com o objetivo de identificar os vírus respiratórios circulantes ${ }^{2,3}$. Assim, todos os casos suspeitos de SRAG são hospitalizados, notificados e investigados para a doença ${ }^{1}$.

Por meio da vigilância epidemiológica da SRAG é possível prevenir e monitorar os casos graves da síndrome, identificar os tipos e subtipos virais circulantes, realizar análise antigênica e genética dos vírus, identificar novos subtipos virais, monitorar a resistência aos antivirais e a demanda de atendimento pela doença e controlar os possíveis surtos, visando a tomada de decisão em saúde pública para sua prevenção e controle ${ }^{4}$.

Em todo o mundo, a circulação dos vírus influenza permanece elevada, com o vírus sazonal A sendo o mais prevalente ${ }^{5}$. O Brasil apresenta o mesmo perfil de circulação, com a maior prevalência do vírus influenza A H1N1 pandêmico 2009 (H1N1pdm09), conforme boletim epidemiológico do Ministério da Saúde ${ }^{6}$. Este cenário mudou recentemente com a pandemia pelo novo coronavírus, que iniciou no final de 2019 na China, e teve seus primeiros casos no Brasil no início de $2020^{7}$.

A SRAG pode evoluir para complicações, que levam ao aumento do risco de internação hospitalar em Unidade de Terapia Intensiva (UTI), com o uso de ventilação mecânica, e inclusive ao óbito $^{8}$. Alguns fatores estão associados a um pior prognóstico, como a presença de doenças crônicas, extremos etários, cepa viral, ausência de vacinação prévia e uso de medicamentos antivirais após 72 horas do início dos sintomas, características que devem ser investigadas ${ }^{9,10}$.

Dessa forma, o objetivo do estudo foi analisar o perfil epidemiológico e os fatores associados à internação e óbitos por SRAG em uma região central do Brasil, entre os anos 2013 e 2018.

\section{Métodos}

Estudo de coorte retrospectiva com análise de dados referente aos casos notificados de SRAG e sua evolução durante a internação no estado de Goiás. Localizado na região Centro-Oeste, ocupa uma área de $340.125 .715 \mathrm{~km}^{2}$, sendo o sétimo estado brasileiro em extensão territorial. Está na oitava colocação do Índice de Desenvolvimento Humano do país (IDH igual a 0,735) ${ }^{11}$.

Todos os casos notificados de SRAG segundo a definição do Ministério da Saúde ${ }^{2}$, no Sinan Influenza Web, residentes no estado de Goiás, independente da faixa etária, foram considerados elegíveis para o estudo. A SRAG é definida, segundo o Ministério da Saúde, como o indivíduo de qualquer idade que apresente quadro de síndrome gripal (febre, tosse ou dor de garganta, e pelo menos um dos seguintes sintomas: cefaleia, mialgia ou artralgia) associado a um quadro de dispneia ou saturação de oxigênio $\mathrm{SpO} 2<95 \%{ }^{2}$.

A coleta dos dados foi realizada em 10 de abril de 2019. Em 2012, houve modificação na ficha de notificação e a vigilância epidemiológica direcionou o monitoramento de influenza por novo subtipo pandêmico para SRAG. Para que as análises fossem conduzidas de forma homogênea com as mesmas variáveis, optou-se por utilizar os dados a partir do ano de 2012. Dessa forma, foram coletados os dados notificados entre os anos de 2013 e 2018.

Este projeto foi aprovado pelo Comitê de Ética em Pesquisa vinculado diretamente ao Centro de Excelência em Ensino, Pesquisa e Projetos "Leide das Neves Ferreira” (GCEEPP-LNF), e ao Comitê de Ética em Pesquisa vinculado ao Hospital das Clínicas da Universidade Federal de Goiás (UFG), e atendeu as condições estabelecidas pela resolução MS/CNS 466/2012.

\section{Análise estatística dos dados}

$\mathrm{Na}$ análise descritiva, foram obtidas frequências absolutas e relativas segundo cada categoria em análise (características sociodemográficas e dados sobre a prevenção, tratamento e evolução da doença). Métodos de análise multivariada dos 
dados foram utilizados para verificar a associação entre as variáveis de exposição e desfechos (óbitos e internação em UTI), sendo consideradas como variáveis de exposição a vacinação prévia, uso de antiviral, presença do vírus Influenza A/B, VSR, outros vírus respiratórios, tempo de tratamento em dias e faixa etária.

Inicialmente foi realizada análise bivariada testando a associação das variáveis de exposição com os desfechos pelo cálculo do Odds Ratio (OR) e valor de p. As variáveis cuja associação com o desfecho apresentou $\mathrm{p} \leq 0,20$ foram incluídas no modelo de regressão logística multivariada. Este modelo foi saturado progressivamente com a adição de cada uma das variáveis, observando-se, para fins de inclusão no modelo definitivo, seus efeitos sobre a precisão do mesmo e sobre o ajuste das demais variáveis. As variáveis consideradas associadas com o desfecho apresentaram, ao final, valor de $\mathrm{p}<0,05$. Para a análise estatística dos dados foi utilizado o Software IBM SPSS Statistics versão 25.0.

\section{Resultados}

Entre 2013 e 2018, foram notificados 4.832 casos de SRAG em Goiás. O maior percentual de notificações ocorreu no sexo feminino $(53,4 \%)$ e na faixa etária de 20 a 59 anos $(45,8 \%)$. Demais dados sociodemográficos estão apresentados na Tabela 1.

Com relação à prevenção, tratamento e evolução da síndrome, somente $20,7 \%$ dos pacientes foram vacinados previamente contra influenza e $70,7 \%$ deles fizeram uso de antiviral. Em 34,6\% dos casos houve internação em UTI e 19\% dos pacientes evoluíram para óbito (Tabela 1).

Em relação à etiologia, observou-se predomínio do vírus influenza $\mathrm{A}$, seguido do VSR em todo período de estudo. Na subtipagem do vírus, ocorreu predomínio do vírus influenza A (H1N1pdm09). Já os casos sem etiologia, definidos como SRAG não especificada, observamos um total de 2.796 pacientes, o que corresponde a aproximadamente $58 \%$ das notificações, com um aumento de notificações nos anos de 2016 e 2018. Nestes mesmos anos, houve também um aumento significativo de casos em comparação com as outras sazonalidades e, consequentemente, do número de internações em UTI e óbitos (Figura 1).

A Tabela 2 apresenta os resultados das análises bivariadas e multivariadas entre as variáveis de exposição com o desfecho óbito. Na análise bivariada observou-se maior chance de óbito nos indivíduos que não utilizaram antiviral, naqueles cuja SRAG foi causada pelo vírus influenza $\mathrm{A}$ ou $\mathrm{B}$, com maior número de dias para o início do tratamento e de idade mais avançada. Na análise multivariada, por sua vez, as variáveis uso de antiviral, infecção por influenza A ou B e por VSR perderam associação com o desfecho, mantendo associação as variáveis tempo de início do tratamento e faixa etária. Observou-se também na análise multivariada maior chance de óbito nos indivíduos que não se vacinaram previamente contra influenza.

Para o desfecho internação em UTI, na análise bivariada, observou-se que o não uso de antiviral, infecção por influenza A ou B, pelo VSR e por outros vírus respiratórios reduziram a chance de internação em UTI, enquanto o maior tempo de início do tratamento aumentou a chance dessa complicação. Na análise multivariada as variáveis infecção por influenza A ou B e por VSR continuaram associadas negativamente com o desfecho enquanto a variável tempo de início do tratamento permaneceu associada positivamente com o desfecho (Tabela 3).

\section{Discussão}

Com este estudo foi possível observar as notificações por SRAG no estado de Goiás por um período de seis anos. Analisando as características sociodemográficas dos casos confirmados, observou-se que não houve diferença significativa em relação ao sexo, ao contrário de estudos realizados na Nova Zelândia, Estados Unidos e França em que o sexo feminino foi predominante ${ }^{12-16}$. Em relação à faixa etária, o maior número de pessoas acometidas estava com idade entre $20 \mathrm{e}$ 59 anos, grupo que não faz parte da indicação de vacinação contra a influenza pelo Ministério da Saúde do Brasil. Alta frequência de casos também foi observada em grupos considerados de maior risco, crianças menores de cinco anos $(28,6 \%)$ e idosos acima de 60 anos (15,0\%), como observado em outros estudos ${ }^{12,17,18}$. É claro na literatura que indivíduos em extremos etários possuem maior risco para quadros respiratórios, como gripe e pneumonia ${ }^{19,20}$.

O Brasil possui um dos maiores programas de imunização do mundo, com um volume muito grande de doses ministradas contra diversas doenças, além de ser gratuito ${ }^{21}$ E dentre essas vacinas está a contra a influenza, incorporada no Programa Nacional de Imunizações (PNI) em 
Tabela 1. Características sociodemográficas dos pacientes notificados por SRAG em Goiás e características relacionadas à prevenção, tratamento $\mathrm{e}$ evolução da doença, 2013-2018 $(n=4.832)$.

\begin{tabular}{|c|c|c|}
\hline Características & $\mathbf{N}$ & $\%$ \\
\hline \multicolumn{3}{|l|}{ Sexo } \\
\hline Masculino & 2250 & $46,6 \%$ \\
\hline Feminino & 2581 & $53,4 \%$ \\
\hline Ignorado & 1 & $0,0 \%$ \\
\hline \multicolumn{3}{|l|}{ Faixa etária } \\
\hline 0 a 5 anos & 1381 & $28,6 \%$ \\
\hline 6 a 19 anos & 512 & $10,6 \%$ \\
\hline 20 a 59 anos & 2213 & $45,8 \%$ \\
\hline 60 anos e mais & 724 & $15,0 \%$ \\
\hline Ignorado & 2 & $0,0 \%$ \\
\hline \multicolumn{3}{|l|}{ Gestação } \\
\hline $1^{\circ}$ trimestre & 47 & $1,0 \%$ \\
\hline $2^{\circ}$ trimestre & 104 & $2,2 \%$ \\
\hline $3^{\circ}$ trimestre & 125 & $2,6 \%$ \\
\hline Idade gestacional ignorada & 5 & $0,1 \%$ \\
\hline Não & 1236 & $25,6 \%$ \\
\hline Não se aplica & 3147 & $65,1 \%$ \\
\hline Ignorado & 168 & $3,5 \%$ \\
\hline \multicolumn{3}{|l|}{ Cor da pele } \\
\hline Branca & 1471 & $30,4 \%$ \\
\hline Negra & 235 & $4,9 \%$ \\
\hline Amarela & 53 & $1,1 \%$ \\
\hline Parda & 2438 & $50,5 \%$ \\
\hline Indígena & 5 & $0,1 \%$ \\
\hline Ignorado & 630 & $13,0 \%$ \\
\hline \multicolumn{3}{|l|}{ Escolaridade } \\
\hline Sem escolaridade & 110 & $2,3 \%$ \\
\hline Fundamental & 809 & $16,7 \%$ \\
\hline Médio & 800 & $16,6 \%$ \\
\hline Superior & 311 & $6,4 \%$ \\
\hline Não se aplica & 1423 & $29,4 \%$ \\
\hline Ignorado & 1379 & $28,5 \%$ \\
\hline \multicolumn{3}{|c|}{ Prevenção, tratamento e evolução } \\
\hline \multicolumn{3}{|c|}{ Vacinação prévia } \\
\hline $\operatorname{Sim}$ & 998 & $20,7 \%$ \\
\hline Não & 2854 & $59,1 \%$ \\
\hline Ignorado & 980 & $20,3 \%$ \\
\hline \multicolumn{3}{|l|}{ Uso de antiviral } \\
\hline Sim & 3416 & $70,7 \%$ \\
\hline Não & 1203 & $24,9 \%$ \\
\hline Ignorado & 213 & $4,4 \%$ \\
\hline \multicolumn{3}{|l|}{ Hospitalização } \\
\hline $\operatorname{Sim}$ & 4797 & $99,3 \%$ \\
\hline Não & 30 & $0,6 \%$ \\
\hline Ignorado & 5 & $0,1 \%$ \\
\hline
\end{tabular}

Tabela 1. Características sociodemográficas dos pacientes notificados por SRAG em Goiás e características relacionadas à prevenção, tratamento e evolução da doença, 2013-2018 ( $\mathrm{n}=4.832)$.

\begin{tabular}{lrr}
\hline \multicolumn{1}{c}{ Características } & N & \% \\
\hline Internação em UTI & & \\
$\quad$ Sim & 1670 & $34,6 \%$ \\
Não & 2969 & $61,4 \%$ \\
$\quad$ Ignorado & 193 & $4,0 \%$ \\
Evolução & & \\
$\quad$ Alta & 3740 & $77,4 \%$ \\
Óbito & 920 & $19,0 \%$ \\
$\quad$ Ignorado & 172 & $3,6 \%$ \\
\hline
\end{tabular}

UTI: Unidade de Terapia Intensiva.

$1999^{22}$, que é disponibilizada gratuitamente para os grupos de risco de todo o país, dentre eles: crianças na faixa etária de seis meses a menores de seis anos de idade, gestantes, puérperas, portadores de doenças crônicas não transmissíveis, entre outros. Foi observado neste estudo que 59,1\% dos indivíduos acometidos por SRAG não receberam vacinação prévia contra influenza. Vale ressaltar que o maior número de casos em nosso estudo se encontra na faixa etária não coberta pela vacinação, oferecida pelo PNI. Mesmo não sendo ofertada a toda população por meio do SUS, a vacinação contra influenza está disponível na rede privada de saúde. É importante destacar que a vacinação contra a influenza, como medida de saúde pública, auxilia na redução das hospitalizações, especialmente em pessoas do grupo de risco (crianças menores de cinco anos, idosos e gestantes $)^{23}$. No entanto, vários fatores influenciam na efetividade da vacina, como a compatibilidade das cepas circulantes com as cepas da vacina, resposta imunológica do indivíduo, vacinação prévia pela mesma cepa da vacina vigente, dentre outros ${ }^{24,25}$.

$\mathrm{Na}$ suspeita da doença, todos os pacientes devem iniciar o tratamento com antiviral o mais precoce possível, mesmo sem a identificação etiológica ${ }^{2}$. A administração do antiviral pode reduzir a duração da sintomatologia, carga viral, bem como a capacidade de transmissão ${ }^{26}$. O não uso do antiviral e/ou o uso inadequado (acima de 48 horas do início dos sintomas) está associado a complicações da infecção pelo vírus, como agravamento seguido de internação em UTI e evolução a óbito ${ }^{8}$, e o não uso do medicamento foi observado em $24,9 \%$ dos pacientes no presente estudo. Dessa forma, é necessário fortalecer as ações para o manejo adequado dos pacientes 


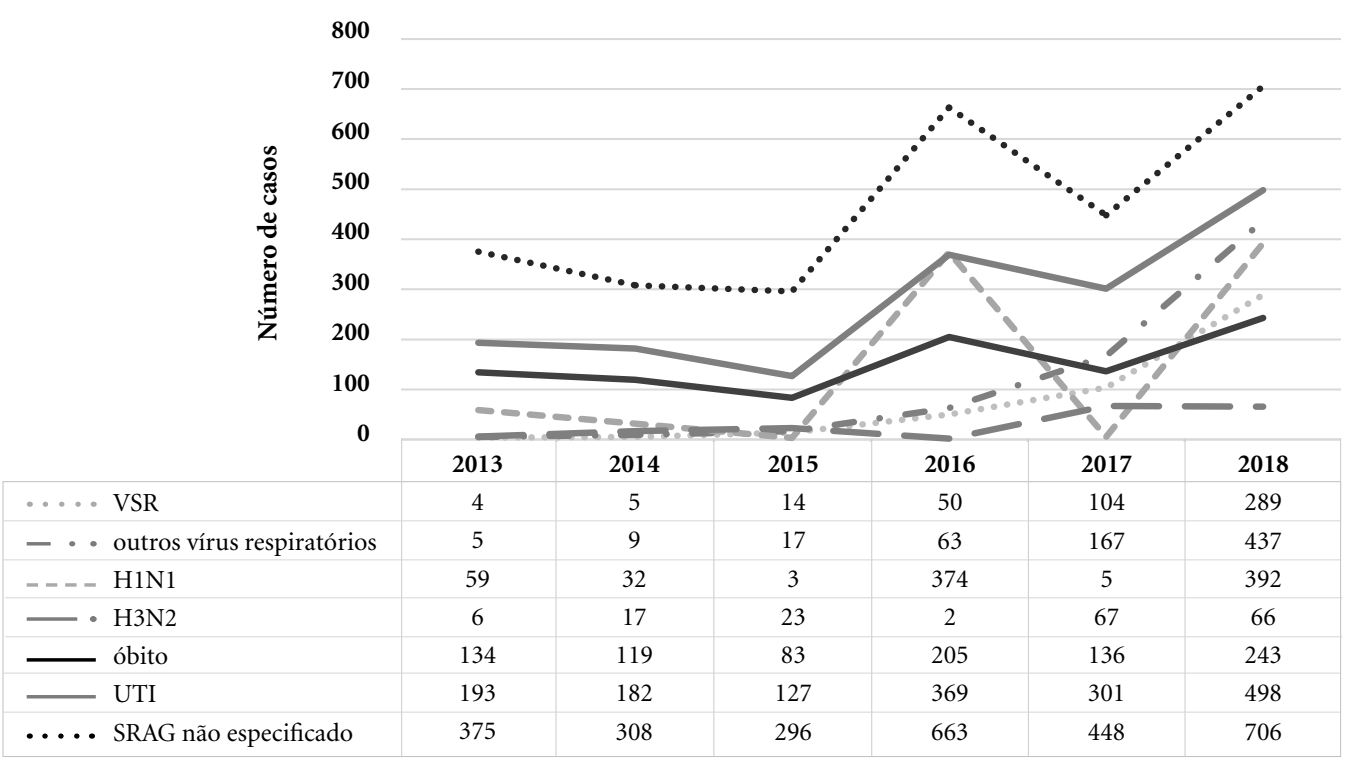

Figura 1. Evolução temporal dos óbitos e internações em UTI por SRAG em Goiás e do número de casos segundo diagnóstico etiológico, 2013-2018.

VSR: Vírus Sincicial Respiratório; UTI: Unidade de Terapia Intensiva.

com SRAG como estratégia de redução dos casos graves desta doença.

Entre os agentes etiológicos foi observado que o vírus influenza é o mais prevalente, seguido do VSR. Entre os vírus Influenza houve maior frequência do vírus A (H1N1pdm09), seguido pelo A (H3N2) e o vírus influenza B. Observou-se variações de ano a ano para ambos os patógenos, que pode ser devido a diferentes cepas e padrões de circulação dos vírus a cada sazonalidade ${ }^{27}$, o que destaca a importância de estimar a carga da doença associada à infecção pelos vírus, bem como fortalecer as ações de vigilância na investigação epidemiológica da síndrome, o que corrobora com as medidas de saúde pública ${ }^{4,28}$. Este mesmo padrão de circulação viral é observado em outros estudos onde os vírus A (H1N1pdm09) representa a maioria dos vírus identificados ${ }^{29-31}$.

Interessante ressaltar que em 2016 e 2018 foi observado aumento significativo dos casos de SRAG no estado de Goiás. Acredita-se que este cenário se deve ao fato de que nos anos anteriores (2015 e 2017), a cobertura vacinal para alguns grupos específicos, como portadores de comor- bidades, ficou abaixo da meta de $90 \%$, favorecendo uma maior circulação viral nas sazonalidades seguintes.

A identificação do vírus influenza em pacientes sintomáticos está associada a um maior risco de complicações respiratórias, representado pelos óbitos e internação em UTI, além de outros fatores contribuírem, como faixa etária do paciente, cepa viral, status vacinal e uso de antiviral ${ }^{27}$. Observou-se, em nosso estudo, que $19 \%$ dos pacientes evoluíram para óbito e 34,6\% internação em UTI, conforme observado em outros estudos ${ }^{8,32}$. Ainda, observou-se que a chance de ter um desfecho grave (óbito e internação em UTI) foi maior em adultos jovens ou idosos, quando comparado com crianças, naqueles que não se vacinaram previamente contra a influenza, e com maior tempo em dias do início do tratamento com o antiviral. Segundo Naudion et al..$^{33}$, este vírus é o mais associado às complicações da síndrome e, da mesma forma, o não uso do antiviral e a ausência de imunização estão relacionados com as complicações ${ }^{33}$. Outros estudos apontam que pacientes que fizeram uso do antiviral e tiveram va- 


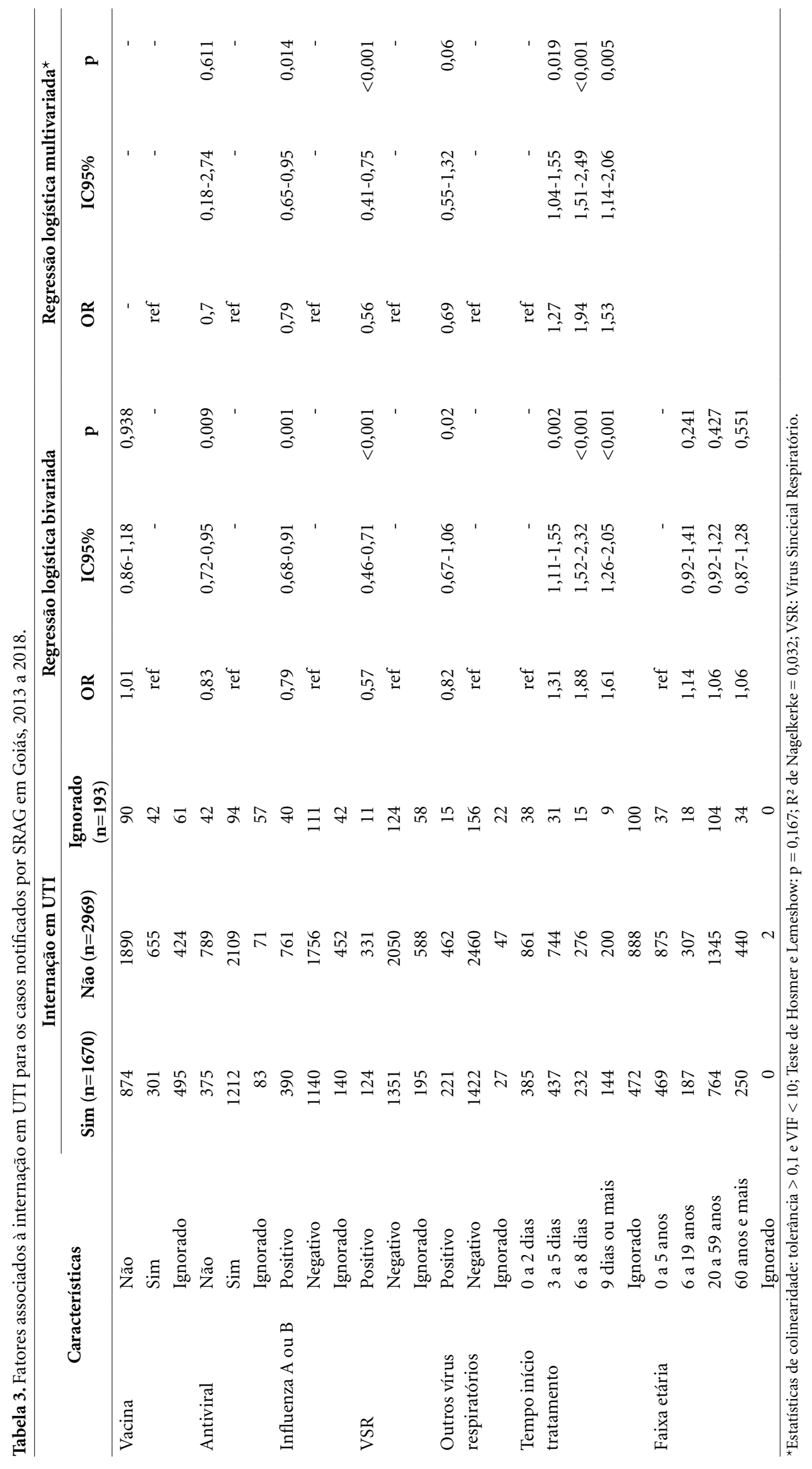


cinação prévia para influenza, apresentaram redução significativa do risco de complicações ${ }^{19,22}$. Em relação a faixa etária, estudos na literatura evidenciaram também maior chance de complicações em idades mais avançadas ${ }^{8,33}$.

Como limitação do estudo, podemos destacar que a frequência dos casos e óbitos por SRAG pode ser subestimada devido a notificação passiva dos mesmos, bem como da sensibilidade dos profissionais de saúde na identificação do caso suspeito. A subnotificação de casos no SINAN não deveria ocorrer, pois a SRAG é de notificação compulsória para fins de vigilância em saúde ${ }^{34}$. A quantidade de casos não identificados, porém capazes de transmitir a doença, é uma característica epidemiológica crítica que modula o potencial de propagação do vírus ${ }^{35}$. Outra limitação é a utilização de dados secundários, que por vezes podem ser incompletos e com limitações de variáveis. Assim, é necessário que os níveis locais de vigilância sejam capacitados e utilizem amplamente os dados dos sistemas de informação, a fim de identificar fragilidades e inconsistências nos dados, melhorando a qualidade dos sistemas e tornando-os efetivos ${ }^{34}$.

\section{Colaboradores}

KLR Araujo e YMF Ternes participaram da concepção, delineamento e redação do artigo. EC Aquino, LLS Silva e YMF Ternes participaram da análise estatística e interpretação dos dados. Todos os autores participaram da revisão crítica e aprovação da versão a ser publicada.
Tem-se no SINAN, assim como em outros Sistemas de Informação em Saúde do Ministério da Saúde do Brasil, uma fonte valiosa de informações em saúde que auxiliam no planejamento de políticas e programas de saúde, colaborando no processo de tomada de decisões, além de permitir que seja avaliado o impacto das intervenções. Dessa forma, mesmo diante das limitações, são ferramentas importantes para a saúde pública e, em especial, para a vigilância epidemiológica dos agravos. Adicionalmente, com a pandemia do novo coronavírus no país em 2020, o sistema de vigilância da SRAG está sendo reforçado, representando um passo importante para a saúde pública no que diz respeito à vigilância epidemiológica e laboratorial dos agentes respiratórios.

\section{Conclusão}

Inúmeros casos de doenças respiratórias provocadas pelo vírus influenza e outros agentes etiológicos ocorrem no estado de Goiás, e foi possível observar que a gravidade da síndrome, caracterizada pela internação em UTI e óbitos, está associada com a idade do paciente, tempo de início do tratamento com o antiviral e o status vacinal. 


\section{Referências}

1. Ribeiro SA, Brasileiro GS, Soleiman LNC, Silva CC, Kavaguti CS. Severe acute respiratory syndrome caused by the influenza A (H1N1) virus. Jornal Bras Pneumol 2010; 36:386-389.

2. Brasil. Ministério da Saúde (MS). Secretaria de Vigilância em Saúde. Departamento de Vigilância das Doenças Transmissíveis. Protocolo de tratamento de Influenza: 2018. Brasília: MS; 2018.

3. Goiás. Sevretaria de Estado da Saúde. Nota Técnica SUVISA/SES-GO. Recomendações referentes à prevenção, vigilância epidemiológica e tratamento da síndrome gripal $(S G)$ e sindrome respiratória aguda grave (SRAG). Goinânia: Superintendência de Vigilância em Saúde; 2018.

4. Brasil. Ministério da Saúde (MS). Secretaria de Vigilância em Saúde. Departamento de Vigilância das Doenças Transmissíveis. Guia para a Rede Laboratorial de Vigilância de Influenza no Brasil. Brasília: MS; 2016.

5. Centers for Diseases Control and Prevention (CDC) Centros de Controle e Prevenção de Doenças, Centro Nacional de Imunização e Doenças Respiratórias (NCIRD) [Internet]. 2020. Disponível em: https:// www.cdc.gov/flu/index.htm

6. Brasil. Ministério da Saúde (MS). Secretaria de Vigilância em Saúde.. Influenza: Monitoramento até a Semana Epidemiológica 49 de 2019. Boletim Epidemiológico 2019; 50:1-43.

7. Brasil. Ministério da Saúde (MS). Boletim Epidemiológico Especial 14. COE-COVID19 [Internet]. 2020 [acessado 2020 Jun 16]. Disponível em: https://portalarquivos.saude.gov.br/images/pdf/2020/ April/27/2020-04-27-18-05h-BEE14-Boletim-doCOE.pdf

8. Coleman BL, Fadel SA, Fitzpatrick T, Tomas SM. Risk factors for serious outcomes associated with influenza illness in high-versus low-and middle-income countries: Systematic literature review and meta-analysisle. Influenza Other Respiratory Viruses 2018; 12(1):22-29.

9. Ribeiro AF, Pellini ACG, Kitagawa BY, Marques D, Madalosso G, Figueira GCN, Fred J, Albernaz RKM, Carvalhanas TRMP, Zanetta DMT. Risk Factors for Death from Influenza A (H1N1) pdm09, State of São Paulo, Brazil. Public Library od Sci 2015; 1-14.

10. Ren Y, Yin Y, Li W, Lin Y, Liu T, Wang S, Zhang SY, Li Z, Wang XJ, Bi ZQ. Risk factors associated with severe manifestations of 2009 pandemic influenza A (H1N1) infection in China: a case- control study. Virology $J$ 2013; 1-7.

11. Brasil. Instituto Brasileiro de Geografia e Estatística (IBGE). Cidades e Estados Brasileiros [Internet]. 2019 [acessado 2020 Jun 16]. Disponível em: https://www. ibge.gov.br/cidades-e-estados/go.html.

12. Delgado-Sanz C, Mazagatos-Ateca C, Oliva J, Gherasim A, Larrauri A. Illness Severity in Hospitalized Influenza Patients by Virus Type and Subtype, Spain, 2010-2017. CDC- Centre for Disease Prevention and Control Emerging Infectious Diseases. CDC 2020; 26(2):2010-2017.
13. Reacher M, Warne B, Reeve L, Verlander NQ, Jones NK, Ranellou K, Cristou S, Wrigth C, Choudhry S, Zambon M, Sander C, Zhang H, Jalal H. Influenza -associated mortality in hospital care: a retrospective cohort study of risk factors and impact of oseltamivir in an English teaching hospital, 2016 to 2017. Revista da Europa sobre vigilância de doenças infecciosas, epidemiologia, prevenção e controle. Euro Surveill 2019; 1-12.

14. Guerrisi C, Ecollan H, Southy C, Rossignol G, Turbelin C, Debin H, Goronflot T, Boelle PY, Hanslik T, Colizza V, Blanchon T. Factors associated with influenza-like-illnes: a crowdsourced cohort study from 2012/13 to 2017/18. BMC Public Health 2019; 879:1-9.

15. Huang QS, Bandaranayake D, Madeira T, Newbern EC, Sements R, Ralston J, Waite B, Bissielo A, Prasad N, Todd A, Jelley L, Gunn W, McNicholas A, Metz T, Lawrence S, Collis E, Retter U, Wong SS, Webby R, Bocacao J, Haubrock J, Mackereth L, Turner N, McArdle B, Cameron J, Reynolds EG, Baker MG, Grant CC, McArthur C, Roberts S, Trenholme A, Wong C, Taylor S, Thomas P, Duque J, Gross D, Thompson MG, Widdowson MA. Risk Factors and Attack Rates of Seasonal Influenza Infection: Results of the Southern Hemisphere Influenza and Vaccine Effectiveness Research and Surveillance (SHIVERS) Seroepidemiologic Cohort Study. J Infect Dis 2019; 219:347-357.

16. Ferdinands JM, Gaglani M, Martin ET, Middleton D, Monto AS, Murthy K, Silveira FP, Talbot HK, Zimmerman R, Alyanak E, Strickland C, Spencer S, Fry AM. Prevention of Influenza Hospitalization Among Adults in the United States, 2015-2016: Results From the US Hospitalized Adult Influenza Vaccine Effectiveness Network (HAIVEN). J Infect Dis Vaccination Against Influenza Hospitalization 2019; 220:20152016.

17. Rabarison JH, Tempia S, Harimanana A, Guillebaud J, Razanajatovo NH, Ratsitorahina M, Heraud JM. Burden and epidemiology of influenza-and respiratory associated severe acute respiratory illness hospitalization in Madagascar, 2011-2016. Influenza Other Respir Viruses 2019; 13(2):138-147.

18. Código SH, Bakken IJ, Blasio BF Haberg SE. Burden of medically attended influenza in Norway 2008-2017. Influenza Other Respir Viruses 2019; 13(3):240-247.

19. Brent SE, Pullenayegum E, Russell ML, Loeb M. Effect of seasonal influenza vaccination on influenza symptom severity among children in Hutterite communities: Follow up study of a randomized trial. Influenza Other Respir Viruses 2020; 14(1):28-36.

20. Lopes NR, Rodrigues BB, Tiago DC, Alvarenga LCR, Medeiros LMM, Ribeiro FAC, Rabahi MF. Factors associated with anti-influenza and anti-pneumococcal vaccination in elderly. Braz J Development 2019; 5(9):15451-15462.

21. Brasil. Ministério da Saúde (MS). Secretaria de Vigilância em Saúde. Departamento de Vigilância Epidemiológica. Programa Nacional de Imunizações (PNI): 40 anos. Brasília: MS; 2013. 
22. Brasil. Ministério da Saúde (MS). Informe Técnico $21^{a}$ Campanha Nacional de Vacinação contra a Influenza. Brasília: MS; 2019.

23. Andrew MK, Shinde V, Ye L, Hatchette T, Haguinet F, Dos Santos L, McElhaney JE, Ambrose U, Boivin G, Bowie W, Chit Um, Elsherif H, Verde K, Halperin S, Ibarguchi B, Johnstone J, Katz K, Langley J, Leblanc J, Loeb M, MacKinnon-D C, McCarthy U, McGeer U, Powis J, Richardson D, Semret M, Stiver G, Trottier S, Valiquette L, Webster D, McNeil SA. The Importance of Frailty in the Assessment of Influenza Vaccine Effectiveness Against Influenza-Related Hospitalization in Elderly People. J Infect Dis 2017; 216:405-414.

24. Chow EJ, Davis CT, Elal AIA, Alabi N, Azziz-Baumgartner E, Barnes J, Blanton L, Brammer L, Budd AP, Burns E, Davis WW, Dugan VG, Fry AM, Garten R, Grohskopf LA, Gubareva L, Jang Y, Jones J, Kniss K, Lindstrom S, Mustaquim D, Porter R, Rolfes M, Sessions W, Taylor C, Wentworth DE, Xu X, Zanders N, Katz J, Jernigan D. Update : Influenza Activity-United States and Worldwide, May 20-October 13, 2018. CDC 2018; 67(42):1178-1185.

25. Flannery B, Chung JR, Belongia EA, Mclean HQ, Gaglani M, Murthy K, Zimermmam RK, Nowalk MP, Jackson ML, Jackson LA, Monto AS, Martin ET, Foust A, Sessões W, Berman L, Barnes JR, Spencer S, Fry AM. Interim Estimates of 2017-18 Seasonal Influenza Vaccine Effectiveness-United States, February 2018. CDC 2018; 67(6):180-185.

26. Cheng HY, Chen WC, Chou YJ, Huang AS, Huang WT. Containing influenza outbreaks with antiviral use in long-term care facilities in Taiwan, 2008-2014. Influenza Other Respir Viruses 2018; 12(2):287-292.

27. Iuliano AD, Roguski KM, Chang HH, Muscatello DJ, Palekar R, Tempia S, Cohen C, Gran JM. Schanzer D, Cowling BJ, Wu P, Kyncl J, Ang LW, Park M, Redlberger-Fritz M, Yu H, Espenhain L, Krishnan A, Emukule G, VanAsten L, Silva PS, Aungkulanon S, Buchholz U, Widdowson MA, Bresse JS. Estimates of global seasonal influenza-associated respiratory mortality: a modelling study. Lancet 2019; 391(10127):1285-1300.

28. Brasil. Ministério da Saúde (MS). Secretaria de Vigilância em Saúde. Coordenação-Geral de Desenvolvimento da Epidemiologia em Serviços. Guia de Vigilância em Saúde: volume único. Brasilia: MS; 2017.

29. Chow EJ, Davis CT, Elal AIA, Alabi N, Azziz-Baumgartne Er, Barnes J, Blanton L, Brammer L, Budd AP, Burns E, Davis WW, Dugan VG, Fry AM, Garten R, Grohskopf LA, Gubareva L, Jang Y, Jones J, Kniss K, Lindstrom S, Mustaquim D, Porter R, Rolfes M, Sessions W, Taylor C, Wentworth DE, Xu X, Zanders N, Katz J, Jernigan D. Update: Influenza Activity-United States and Worldwide, May 20-October 13, 2018. US Department of Health and Human Services/Centers for Disease Control and Prevention. CDC 2019; 67(42):2-9.
30. Epperson S, Davis CT, Lynnette B, Elal AIA, Ajayi N, Barnes J, Budd AP, Burns E, Daly P, Dugan VG, Fry AM, Jang Y, Johnson SJ, Kniss K, Kondor R, Grohskopf LA, Gubareva L, Merced-Morales A, Sessions W, Stevens J, Wentworth DE, Xu X, Jernigan D. Update: Influenza Activity-United States and Worldwide, May 19-September 28, 2019, and Composition of the 2020 Southern Hemisphere Influenza Vaccine. US Department of Health and Human Services/Centers for Disease Control and Prevention. CDC 2019; 68.

31. Xu X, Blanton L, Elal AIA, Alabi N, Barnes J, Biggerrstaff M, Brammer L, Budd AP, Burns E, Cummings CN, Garg S, Kondor R, Gubareva L, Kniss K, Nyanseor S, O'Halloran A, Rolfes M, Wendy S, Dugan VD, Fry AM, Wentworth DE, Stevens J, Jernigan D. Update: Influenza Activity in the United States During the 201819 Season and Composition of the 2019-20 Influenza Vaccine. US Department of Health and Human Services/Centers for Disease Control and Prevention. CDC 2019; 68(24):544-551.

32. Pivette M, Nicolay N, Lauzun V, Hubert B. Characteristics of hospitalizations with an influenza diagnosis, France, 2012-2013 to 2016-2017 influenza seasons. Influenza Other Respir Viruses 2020; 14(3):1-9.

33. Naudion P, Lepiller Q, Boullier K. Risk factors and clinical characteristics of patients with nosocomial influenza A infection. J Med Virol 2020; 92(8):10471052.

34. Rosetto EV, Luna EJA. Database linkage for surveillance of the influenza A (H1N1) pdm09 pandemic in Brazil, 2009-2010. Cad Saúde Pública 2016; 32(7):e00014115.

35. Nogueira AL, Nogueira CL, Zibetti AW, Roqueiro N, Bruna-Romero O, Carciofi BAM. Estimativa da Subnotificação de Casos da COVID-19 no Estado de Santa Catarina. Joinville: Universidade Federal de Santa Catarina; 2020.

Artigo apresentado em 19/04/2020

Aprovado em 10/08/2020

Versão final apresentada em 12/08/2020 